\title{
LA PROBLEMÁTICA DE LOS JUEGOS DE PALABRAS EN LAS TRADUCCIONES DE EL LAZARILLO DE TORMES A LA LENGUA INGLESA
}

\author{
BEATRIZ MARÍA RODRÍGUEZ RODRÍGUEZ \\ Universidad de Santiago de Compostela
}

\section{RESUMEN}

Los juegos de palabras constituyen una característica esencial del estilo de $E l$ Lazarillo de Tormes. En este trabajo se han analizado los recursos manejados en relación con esta figura retórica por los traductores a la lengua inglesa en las diecisiete traducciones publicadas hasta la fecha. Se ha prestado especial atención a los factores que han originado el elevado número de omisiones de estos juegos. Un estudio más pormenorizado de algunas traducciones recientemente publicadas ha permitido valorar los parámetros que han condicionado las decisiones de cada traductor.

Palabras claves: juegos de palabras, traducción, Lazarillo

\begin{abstract}
Puns involve an essential feature concerning the style of El Lazarillo de Tormes. This paper aims at analysing the devices and strategies used in the rendering of puns in the seventeen translations published in English. Reasons for the great number of omissions have been closely analysed. Factors affecting the translators' decisions have been highlighted, mainly after an exhaustive analysis of several current translations.
\end{abstract}

Key words: puns, translation, Lazarillo

\section{INTRODUCCIÓN}

Este estudio pretende analizar y comparar las estrategias y técnicas usadas por los traductores a la hora de enfrentarse a los juegos de palabras. Para ello se analizarán estas figuras retóricas en las traducciones a la lengua inglesa de la novela picaresca El Lazarillo de Tormes. Se intentará descubrir los factores que condicionaron las decisiones de los traductores. 
Indudablemente, los juegos de palabras constituyen una figura retórica determinante del estilo de El Lazarillo de Tormes. Se trata de un estilo sumamente elaborado (Blecua 1982: 42) aunque el autor anónimo pretende que no lo parezca. La propia esencia del juego de palabras encierra obvias dificultades que se incrementan en la traducción. Los juegos de palabras no sólo implican un juego de significado, sino también de conceptos y de imágenes culturales. ${ }^{1}$ Encierran fenómenos de polisemia, homonimia, ritmo, gramática, sintaxis, juegos de pronunciación, nombres, referencias culturales, etc. Joan Verdegal (2000) afirma que se trata incluso de un fenómeno de distorsión, de una manipulación. Los problemas de traducción llevan a la utilización de diversos recursos entre los que destacan el préstamo, la expansión, la substitución, y la compensación (Landers 2001: 79-95), en un intento de lograr un efecto equivalente en la lengua meta. Sin embargo, en algunos casos se reconoce la total imposibilidad de traducirlos (Landers 2001: 79), lo que se suele comentar en nota explicativa. Desgraciadamente, también es verdad que en demasiados casos se opta por la omisión total o parcial del juego de palabras sin causa aparente.

En este trabajo se ha analizado en detalle cómo se han traducido cuarenta y cuatro juegos de palabras de El Lazarillo de Tormes en las diferentes traducciones a la lengua inglesa publicadas hasta la actualidad. Muchos de los ejemplos analizados son de carácter repetitivo, y se basan en un juego entre dos formas verbales o dos categorías gramaticales. Como era de esperar, la perspectiva cronológica ha permitido comprobar la evolución de la práctica de la traducción en cuanto a esta figura retórica.

\section{EL PAPEL DE LOS JUEGOS DE PALABRAS EN LAS TRADUCCIONES DE EL LAZARILLO DE TORMES}

En primer lugar cabe destacar que existen diecisiete traducciones diferentes de $E l$ Lazarillo de Tormes a la lengua inglesa, obviando las diversas reediciones, que no se han considerado al no implicar ninguna diferencia que pudiese alterar los resultados de este estudio. Los traductores son los siguientes: David Rowland (Londres 1586); Thomas Roscoe (Londres 1832); Sir Clements Markham (Londres 1908); Louis How (Nueva York 1917); Mariano Lorente (Boston 1924); J. Gerald Markley (Nueva York 1954); Mark Hendricks Singleton (Nueva York 1957); Harriet de Onis (Nueva York 1959); J. M. Cohen (Suffolk 1962); W. S. Mervin (Nueva York 1962); James Parsons (Nueva York 1966); Michael Alpert (Londres 1969); Robert S. Rudder (Nueva York 1973); Stanley Appelbaum (Nueva York 2001). A esto hay que añadir tres traducciones anónimas publicadas en Londres en 1688,1724 y 1789.

Después de un análisis pormenorizado de estas traducciones se ha comprobado una tendencia a la omisión total o parcial de la mayoría de los juegos de palabras analizados. En pocos casos se mantiene el mismo juego mediante traducción literal de los términos fuente, o la utilización de modulaciones logrando un efecto equivalente. La tabla 1 refleja los resultados del análisis.

\footnotetext{
${ }^{1}$ Para un detallado estudio y clasificación de los juegos de palabras véase Walter Redfern (1984) y Dirk Delabatista (1996, 1997).
} 


\begin{tabular}{||c|c|c|}
\hline TRADUCTOR & SE MANTIENE & SE OMITE \\
\hline \hline Rowland (1586) & $9,09 \%$ & $90,90 \%$ \\
\hline Anónimo (1688) & $2,27 \%$ & $97,72 \%$ \\
\hline Anónimo (1726) & ----- & $100 \%$ \\
\hline Anónimo (1789) & $2,27 \%$ & $97,72 \%$ \\
\hline Roscoe (1832) & $2,27 \%$ & $97,72 \%$ \\
\hline Markham (1908) & $9,09 \%$ & $90,90 \%$ \\
\hline How (1917) & $63,63 \%$ & $36,36 \%$ \\
\hline Lorente (1924) & $20,45 \%$ & $79,54 \%$ \\
\hline Markley (1954) & $27,27 \%$ & $72,72 \%$ \\
\hline Singleton (1957) & $9,09 \%$ & $90,90 \%$ \\
\hline Onis (1959) & $25 \%$ & $75 \%$ \\
\hline Cohen (1962) & $22,72 \%$ & $77,27 \%$ \\
\hline Mervin (1962) & $25 \%$ & $75 \%$ \\
\hline Parsons (1966) & $52,72 \%$ & $79,54 \%$ \\
\hline Alpert (1969) & $20,45 \%$ & $75 \%$ \\
\hline Rudder (1973) & $25 \%$ & \\
\hline Appelbaum (2001) & & $76,81 \%$ \\
\hline
\end{tabular}

Tabla 1

Una primera valoración de estos datos permite delimitar en primer lugar la importancia del factor histórico a la hora de analizar una traducción. Como se puede observar en la tabla 1 las omisiones son generalizadas en las traducciones publicadas antes del siglo XX, mientras que su incidencia aumenta a partir de 1917. La práctica de la traducción en cada época parece condicionar en gran medida este aspecto ${ }^{2}$. Las características más relevantes de cada traducción son esenciales a la hora de delimitar otros parámetros que pudieron haber influido en las traducciones de los juegos de palabras.

2 El factor histórico es uno de los más relevantes a la hora de analizar una traducción o de evaluarla (Brunette 2000: 178). 
La traducción de David Rowland es la primera traducción publicada en lengua inglesa, destacando principalmente por su especial papel en el desarrollo de la novela inglesa y su incomparable recepción, avalada por el considerable número de reediciones publicadas hasta la actualidad. Merece la pena subrayar la influencia de una traducción francesa previa realizada por Jean Saugrain $(1561)^{3}$. Rowland utiliza como base de su traducción tanto la traducción francesa como el texto español de Amberes de 1554, aunque no lo hace de forma sistemática. El análisis de las traducciones permite concluir que Rowland sigue un texto u otro de forma arbitraria. El traductor mantiene algunas omisiones y expansiones de la traducción francesa, lo que ha podido desembocar en la omisión de juegos de palabras. A esto hay que añadir que en la época isabelina los traductores solían recurrir a estas estrategias para embellecer el texto según su propio punto de vista (Luttikhuisen 1997: 179). El objetivo del traductor inglés ${ }^{4}$, en clara relación con el propósito del traductor francés, permite también entender la poca importancia dada a los juegos de palabras. Rowland ignora gran parte de los juegos utilizando modulaciones en uno de los términos con la intención de mantener su significado. El traductor afirma que pretende proporcionar: "a true description of the nature $\&$ disposition of sundrie Spaniards. So that by reading hereof, such as haue not traualied Spaine, may as well discerne much of the maners \& customs of that countrey" (1586: 3 ).

En cuanto a las traducciones anónimas la casi completa omisión de estos juegos parece estar en consonancia con la mala calidad de los tres textos. Las omisiones de párrafos e incluso de páginas completas son la tónica dominante en la traducción de 1688, mientras que las expansiones abundan en el texto de 1726, y sobremanera en la traducción de 1789. De hecho, en los tres casos es difícil reconocer el texto fuente. La práctica de la traducción en ese momento y la influencia de las traducciones francesas parecen estar en el origen del gran número de expansiones. En esta misma línea se sitúa la traducción de Roscoe de 1832, en la que se aprecian nuevamente omisiones de páginas completas, lo que implica la total omisión de los juegos de palabras, restándole nuevamente importancia a la figura retórica en sí.

La traducción de How (1917) parece marcar un importante punto de diferencia con las traducciones previas. Por primera vez se observa una clara preferencia por intentar mantener los juegos de palabras. Este hecho parece estar en consonancia con las palabras de Charles Philip Wagner, autor de la introducción de la traducción, donde afirma que "Mr. How's translation will be found to be even more faithful than Rowland, and far superior in other respects to any version in England" (How 1917: xliv). No hay que olvidar que el concepto de fidelidad a principios del siglo XX se identifica con la traducción literal. Esta parece ser la tónica general de la traducción. De hecho, en los casos en los que se mantienen los juegos de palabras se sigue una traducción literal de los términos fuente.

Siguiendo con el análisis cronológico de los datos se puede observar que a partir de la publicación de la traducción de How (1917), y exceptuando la traducción de Singleton (1957), el porcentaje de juegos de palabras mantenidos en el texto meta aumenta de forma

\footnotetext{
${ }^{3}$ Esta traducción se publicó en Paris con el título L'historie plaisante et facetieuse du Lazare de Tormes Espagnol, editada por Ian Longis \& Robert le Mangnier.

${ }_{4}$ Evidentemente, el propósito de una traducción condiciona gran parte de las decisiones del traductor (Brunette 2000: 176).
} 
notable, situándose por encima del $20 \%$ en la mayoría de los casos. Se puede concluir, por tanto, que se empieza a observar una cierta preocupación por considerar el estilo del texto fuente en consonancia con los cambios en la teoría y práctica de la traducción del siglo XX. Evidentemente, la traducción de juegos de palabras requiere un gran esfuerzo que pone a prueba la capacidad de un traductor. Estos datos han llevado a un posterior análisis más detallado de algunas traducciones recientemente publicadas para intentar determinar los factores que condicionaron las decisiones de los traductores. Así, en la traducción de James Parsons (1966) se observa nuevamente un destacado interés por mantener los juegos de palabras del estilo del texto fuente. La traducción de Michael Alpert (1969) es la última publicada en Londres hasta la fecha y una de las más conocidas al formar parte de la colección de clásicos de Penguin. El texto de Robert Rudder (1973) destaca por su recepción, ya que existen varias reediciones a pesar de que se ha publicado recientemente. A esto hay que añadir que forma parte del proyecto Gütenberg de textos electrónicos ${ }^{5}$. Finalmente, la traducción de Stanley Appelbaum (2001) es la última traducción publicada hasta la fecha y la única que recoge tanto el texto fuente como el texto meta. Nos centraremos, por tanto, en el análisis de las traducciones de How, Parsons, Alpert, Rudder y Appelbaum.

Las características de cada una de estas traducciones permiten entender los factores que pudieron condicionar la traducción de los juegos de palabras como el lector al que va dirigida la traducción, el propósito del texto, la influencia de traducciones previas, y las notas a pie de página.

En primer lugar destaca que la característica central de la traducción de Appelbaum reside en que se trata de una "dual edition". Es la primera vez que una traducción recoge paralelamente tanto el texto fuente como el texto meta ${ }^{6}$. Este interés en que el lector tenga el mayor número posible de detalles afectará positivamente a la traducción de los juegos de palabras .

La traducción de Alpert se publica conjuntamente con la traducción realizada por él mismo de la novela de Francisco Quevedo El Buscón. En este caso cabe destacar el tipo de lector al que va dirigida la traducción, ya que la misma se publica en la colección de clásicos de la editorial Penguin. El amplio sector al que va dirigida esta colección podría justificar la escasa importancia dada a los juegos de palabra del texto fuente, ya que existe una preferencia porque el lector siga el hilo narrativo de toda la historia en detrimento de los detalles y del estilo original. Sin embargo, el propio Alpert afirma pretender "to produce for English readers of today the impression they will have made on Spanish readers of the time" (1969: 15). Además menciona expresamente los problemas causados por las traducciones de los juegos de palabras, pero mantiene su especial relevancia en El Buscón mientras que constata su práctica inexistencia en El Lazarillo. Alpert afirma:

\footnotetext{
Véase la página oficial del proyecto: <http://gutenberg.net>

6 Existe una traducción de similares características publicada en el año 2000 (Keith Whithlock, ed. El Lazarillo de Tormes. Warminster: Aris and Philips). Esta traducción recoge también tanto el texto fuente como el texto meta, pero no se trata de una nueva traducción sino de un reedición de la primera traducción a la lengua inglesa, la traducción de David Rowland (1586).
} 
The translator must reproduce these stylistic mannerisms in modern English, if he wants to convey the impression of the original. Present day English is normally much more concise than even modern Spanish, and the temptation is always to simplify. But I have fought against it, and if the translation is wordy in some places so is the original, I have also tried hard to convey the sense of puns (1969: 5).

El análisis de la traducción deja en tela de juicio la objetividad de estas palabras, porque se aprecia que el traductor prefiere mantener el significado y la narración en cuestión. De hecho, coincido con Peter France al declarar que "Alpert's version... is reliable but insipid" (2000: 42).

En cuanto a la traducción de Rudder, éste reitera en la introducción su intento por mantener los juegos de palabras, puesto que: "style is another point of great importance to this novel, principally in the use of conceits" (1973: xiv). El análisis de su traducción permite corroborar esta afirmación. De todas formas, aunque el porcentaje de juegos palabras mantenidos, no es muy elevado un $25 \%$, sí se observa a lo largo de todo el texto una preferencia por mantener el mayor número de detalles posibles, y lo hace sin recurrir a ningún tipo de anotación.

En este sentido, las notas a pie de página son un recurrido recurso al poder proporcionar relevantes detalles sobre los juegos de palabras. En el caso de las traducciones que estamos analizando con más detalle sólo las traducciones de Parsons y Appelbaum incluyen notas ${ }^{7}$. Parsons incluye 220 notas finales que recogen todo tipo de comentarios culturales e incluso referencias a problemas de traducción planteados o a pasajes de imposible traducción. A esto hay que añadir que Appelbaum incluye 89 notas a pie de página, notas que en ciertos casos, como en la traducción de Parsons, jugarán un papel importante en cuanto a los juegos de palabras. El propio Appelbaum explica que: "Another feature of the original text is its unflagging humour frequently taking the form of puns and word plays. Whenever possible the English translator offers an equivalent but when this was although impossible, or the meaning would have had to be unduly strained, the word play is pointed out in a footnote" (2001: xiv).

Estas notas junto con el hecho de que se trata de una edición que recoge el texto fuente y meta implicando un notable interes para eruditos e investigaciones, podría explicar el interés por mantener los juegos de palabras como rasgos de estilo.

\section{EJEMPLOS DE JUEGOS DE PALABRAS}

El análisis de las traducciones de algunos pasajes permiten corroborar estas afirmaciones y extraer nuevas conclusiones.

En la mayoría de los ejemplos se observan juegos de palabras entre diferentes categorías léxicas con lo que se acentúa el tono repetitivo. Las dificultades lingüísticas que se plantean en la traducción son obvias.

\footnotetext{
${ }^{7}$ Es cierto que la traducción de How incluye notas, concretamente cien, pero las escribió Charles Philip Wagner, la persona que prologó la traducción y no el propio traductor por lo que he decidido no considerarlas al no ser un recurso del traductor para clarificar algún punto en relación con los juegos de palabras
} 
[1] la longaniza había pringado y comídose las pringadas (Rico 2000: 38$)^{8}$

he had basted the sausage and eaten the bastings (How 1917: 24)

the sausage had begun to drip and he had eaten the drippings (Parsons 1966: 27)

When I had basted the sausage and he had eaten the savoury grease (Alpert 1969: 33)

When the sausage had been basted and he had sopped up and eaten the drippings with a piece of bread (Rudder 1973: 19)

the grease had oozed from the sausage and we had eaten bread smeared with the grease (Appelbaum 2001: 38)

En el ejemplo 1 a los problemas lingüísticos se añade un problema cultural. El juego sólo lo mantienen How, Parsons y Appelbaum, aunque utilizan términos diferentes. El significado se mantiene con más o menor detalle en todos los casos. Este ejemplo refleja la base de las traducciones de Alpert y Rudder, es decir, la preferencia por mantener el significado pero de forma escueta en el primer caso, y de proporcionar el mayor número de detalles posibles en el segundo.

[2] “¿Qué es esto, Lazarillo?” "Lacerado de mî” (Rico 2000: 39)

'What is this Lazarillo?' 'Poor Lazaro' said I (How 1917: 25)

'What's the meaning of this, Lazarillo?' 'Lacerated, you mean' (Parsons 1966: 27)

'What is this Lazarillo?' 'What are you starting one me for?'(Alpert 1969: 25)

'What is this Lazarillo?' 'You mean, 'Lacerated» (Rudder 1973: 19)

'What is this Lazarillo?' 'Woe is me' (Appelbaum 2001: 23)

En el ejemplo 2, a pesar de las dificultades lingüísticas y semánticas que encierra el término "lacerado", How mantiene el juego mediante la utilización del nombre propio en cuestión acompañado por el adjetivo "poor". Esta expansión le permite lograr una traducción bastante apropiada al mantener tanto el juego como el significado. Parsons y Rudder intentan mantener el juego utilizando la misma traducción, una traducción literal. Parsons (1966: 82) incluye una nota en la que deja constancia del juego de palabras.

Existen ejemplos en los que se observa una mayor preferencia por omitir estos juegos que implican una repetición entre diferentes categorías gramaticales. Sin embargo, se trata de casos que no encierran tantas dificultades de traducción. Generalmente se traduce literalmente uno de los términos, mientras que se utiliza una modulación o una expansión en la traducción del segundo, en un intento de mantener el significado.

[3] El aguacil dijo a mi amo que era falsario y las bulas que predicaba eran falsas (Rico 2000: 116)

the constable told my master that he was a forger and the bulls he preached were forged (How 1917: 100)

the constable said that my master was a fake and the documents he proclaimed were false

(Parsons 1966: 64)

the constable said he was a forger (Alpert 1969: 68)

My master was a swindler and that all the pardons he was selling were counterfeit (Rudder 1973: 83)

\footnotetext{
${ }^{8}$ Desde mi punto de vista la edición de Francisco Rico es la más completa y detallada.
} 
the constable called my master a counterfeiter and said that the indulgences he preached were fakes (Appelbaum 2001: 83)

En el ejemplo 3 sólo How y Parsons mantienen el juego de palabras entre el nombre y el adjetivo. En los demás casos se opta por la utilización de una modulación con la consiguiente pérdida de la figura retórica.

Existe un considerable número de ejemplos en los que se ha optado por no mantener el juego de palabras a pesar de que no implique especiales dificultades de traducción. Una modulación o una expansión explicativa suelen ser los recursos manejados por los traductores. Por ejemplo el juego de la frase: "le conoscí ser estranjero, por el poco conocimiento y trato que con los naturales della tenía" (Rico 2000: 98) se omite en todas las traducciones, mientras que en el caso de: "mi amo muy enojado. Y después que los huéspedes y vecinos le hubiesen rogado que perdiese el enojo y se fuese a dormir" (Rico 2000: 116) el juego de palabras sólo se mantiene en la traducción de How mediante los términos: "angry" y "anger" (1917: 100).

En clara oposición existen ejemplos, si bien es verdad que su número es realmente escaso, en los que el juego de palabras se mantiene en la práctica totalidad de estas traducciones. El juego "Moría mala muerte" (Rico 2000: 59) se mantiene utilizando los términos "dying" y "death" en todas las traducciones, excepto en la de Alpert, donde se omite como en la mayoría de los ejemplos en esta traducción: "I suffered horribly" (1969: 42).

Como cabría suponer existen casos en los que se produce la omisión total o parcial en la práctica totalidad de las traducciones debido a las dificultades, o incluso a la imposibilidad de traducción. Consideremos las frases: "sacando no por tasa pan, mas buenos pedazos, torreznos y longaniza. Y ansí buscaba conveniente tiempo para rehacer, no la chaza, sino la endiablada falta que el ciego me faltaba" (Rico 2000: 29). Este juego se omite completamente en todas las traducciones debido a las connotaciones lingüísticas y culturales que encierra el término "chaza". En este pasaje Lázaro se las ingenia para robar pequeños trozos de pan que su amo guarda bajo llave en un arca, lo que se compara con los puntos conseguidos en un juego de pelota. El término "falta" se refiere a un fallo en dicho juego. Parece imposible mantener el juego o encontrar una equivalencia en la lengua meta, aunque también es cierto que no es relevante que el lector tenga estos detalles. Sólo Appelbaum se preocupa por justificar la omisión total del juego en una nota en la que comenta la imposibilidad de traducirlo y su consiguiente pérdida.

El ejemplo 4 recoge un tipo diferente de juegos, un juego de significados originado por una diferencia ortográfica.

[4] "Nueve quedan y un pedazo". "Nuevas malas te dé Dios" (Rico 2000: 58)

'Nine remain and one piece'. 'God send thee nine new pieces of trouble' (How 1917: 42)

'Nine remain, and a piece'. 'Nine misfortunes God give you' (Parsons 1966: 35)

'There are nine loaves and a bit left! 'I hope God gives you the pox nine times!' (Alpert 1969: 42)

'There are nine and a half in there now'. 'May God send you nine pieces of bad news' (Rudder 1973: 38)

'There are nine left and a piece'. 'May God send you bad tidings' (Appelbaum 2001: 37) 
Curiosamente, a excepción de Appelbaum, todos los traductores optan por dejar constancia de alguna manera del juego de palabras mediante la repetición total del primer término pero errando en cuanto al significando. Muy acertadas son en este sentido las notas. Parsons explica: "I have preferred to retain the play on words and sacrifice meaning" (1966: 84), mientras que Appelbaum afirma: "The Spanish has an untranslatable word play" (2001: 37).

Un juego de características similares se recoge en el ejemplo 5. Una diferencia ortográfica conduce a una diferencia en género que implica dos significados. La inexistencia de masculino y femenino en la lengua inglesa dificulta la traducción.

[5] Los dichos clérigos eran de los reverendos, digo que más con dineros que con letras y con reverendas se ordenan (Rico 2000: 114)

those that are ordained more with money than with letters and orders (How 1917: 98)

these clerics were among those reverends, I mean those who are ordained more by money than by studies and episcopal appointment (Parsons 1966: 63)

The clergy were just reverends and, because they had more money than education, had been ordained without following a proper course (Alpert 1969: 67)

These clerics were "appointed reverends" - I mean that they bought their way into the priesthood instead of by going through school (Rudder 1973: 81-82)

those priests were "reverend" (that is money rather than learning had gained in their position, with letters beginning "Reverend sir") (Appelbaum 2001: 83)

En este caso se juega con la referencia a las cartas de recomendación necesarias para que los clérigos se conviertan en reverendos. Appelbaum logra mantener el juego gracias a la expansión: "with letters". Además en nota a pie de página todavía proporciona más detalles sobre el término "reverendas". How mantiene cierto paralelismo realizando una traducción literal. En los demás casos se opta por traducir literalmente el primer término, para utilizar una paráfrasis explicativa en el segundo que, a pesar de las diferencias, parece recoger de forma acertada el significado en todas las traducciones.

En otro orden de cosas, cabe señalar que muchos juegos de palabras entre formas verbales se pierden quizás debido a las características inherentes de la lengua inglesa. Se utiliza con frecuencia el auxiliar en la traducción de la segunda forma verbal. Esto sucede en todos los casos en las traducciones de las frases: "Llevar a la posada con que él lo pasase yo lo pasaba mal" (Rico 2000: 91) y "Los astutos usan como digo el día de hoy, de lo que yo usaría" (Rico 2000: 106). Sin embargo existen algunos ejemplos en los que sí se respeta la repetición. El juego: "sentí lo que sentía" (Rico 2000: 89) se mantiene como "I felt what he was feeling" en los textos de How (1917: 75), Appelbaum (2001: 63), y Parsons: "I have suffered what he was suffering" (1966: 53).

Curiosamente existen casos en los que el juego entre formas verbales se mantiene en todas las traducciones. Por ejemplo, "el cruel ciego ahorraría de mí, quise yo ahorrar dél" (Rico 2000: 33) se traduce siempre utilizando el verbo "to get rid of". Se trata de un caso que no plantea serias dificultades, pero es interesante que todos los traductores optaran por la misma traducción. Lo mismo sucede en el ejemplo "viniese a topar con quien no solo no me mantuviese, mas a quien yo había de mantener” (Rico 2000: 91), aunque en 
este caso los traductores utilizan términos diferentes. Este hecho, que también se aprecia en otros ejemplos, lleva a hablar de "intertextualidad" (Rabadán 1991: 207) o influencia de ediciones previas. Es obvio que los traductores disponían de bastantes traducciones previas que podían consultar, incluso en otros idiomas. Sabemos que Rudder consultó ediciones anteriores (comunicación personal), y Appelbaum afirma que consultó dos traducciones anteriores, aunque tampoco especifica de cuáles se trata (2001: xiv).

Para finalizar, podemos decir que existen dos ejemplos de polisemia de similares características.

[6] hallóse en frío con el frío nabo (Rico 2000: 39)

he found himself chilled by the chilly turnip (How 1917: 25)

he found himself face to face with the cold turnip (Parsons 1966: 27)

He got a shock and a mouthful of cold turnip (Alpert 1969: 34)

he was suddenly stopped cold by the taste of the cold turnip (Rudder 1973: 19)

he remained dumbfounded with that dumb turnip (Appelbaum 2001: 23)

A pesar de las dificultades, el juego del ejemplo 6 se mantiene en prácticamente todas las traducciones, excepto en las de Alpert y Parsons. Alpert, siguiendo la línea de su traducción, prefiere utilizar una modulación. Parsons añade una nota (1966: 82) en la que recoge una traducción literal del texto fuente y la pérdida del juego de palabras por dificultades de traducción.

\section{CONCLUSIONES}

El análisis de los juegos de palabras en estas traducciones permite extraer algunas conclusiones. En primer lugar destaca la relevancia del factor histórico. Se ha demostrado que la práctica de la traducción en la época en que se escribe el texto condiciona en gran medida, como cabría esperar, la técnica de traducción. Antes del siglo XX se aprecia una tendencia a la omisión total o parcial de estas figuras. Se prefiere mantener el significado, la narración propiamente dicha, o se ignora completamente el juego de palabras del texto fuente. De todas formas el considerable número de omisiones y expansiones común a la mayoría de las traducciones de ese momento contribuye a enfatizar este hecho. A comienzos del XX se observa una cierta preferencia por intentar mantener esta figura retórica. La traducción de How de principios de siglo está en clara consonancia con la vuelta a las traducciones fieles, a las traducciones literales. How sigue una traducción literal, quizás incluso demasiado lingüística ignorando la práctica totalidad de las referencias culturales, por lo que se podría entender que mantenga el 63'63\% de los juegos de palabras. Esta cierta preocupación por mantener el estilo original a partir de ese momento ha llevado a buscar otras causas para las traducciones recientemente publicadas. Un análisis detallado de los datos permite comprobar también que no se trata meramente de solventar una dificultad cultural o lingüística, ya que existen recursos para mantener muchos de los juegos pero los traductores no los utilizan.

El propósito del traductor parece ser otro de los factores que más influyen a la hora de traducir los rasgos del estilo del texto fuente. Este factor está claramente relacionado con 
el lector al que va dirigida la traducción. La traducción de Alpert es la que mantiene menos juegos, quizás al tratarse de una edición muy comercial que intenta abarcar un campo de lectores realmente amplio y menos preocupado por los detalles. Un cierto aumento en el porcentaje de juegos de palabras mantenidos en la traducción de Rudder se puede entender desde la perspectiva de la preocupación del traductor por proporcionar el mayor número de detalles posible. A pesar de que no se trata de una traducción anotada, Rudder intenta incluir todas las explicaciones en el texto de la traducción de forma bastante apropiada. En cuanto a las dos traducciones que incluyen notas, podemos comentar que por sus características la traducción de Appelbaum resulta especialmente adecuada para investigadores. Tanto la inclusión de notas a pie de página como el hecho de haber recogido el texto fuente permite una visión completa del proceso de traducción. El elevado porcentaje de juegos de palabras mantenidos (43'18\%), las expansiones explicativas y notas contribuyen a demostrar la capacidad traductora de su autor. Esta misma preocupación de que el lector tenga todos los detalles culturales y lingüísticos se aprecia también en la traducción de Parsons, como demuestra el hecho de que mantenga casi el 53\% de los juegos de palabras. La importancia de las notas queda, por tanto, de relieve en ambas traducciones. Para finalizar quisiéramos decir que se ha observado también la existencia de la intertextualidad o posible influencia de traducciones previas. Curiosamente las traducciones recientemente publicadas con mayor número de juegos de palabras se han publicado en Nueva York. También se ha comprobado que no siempre se pueden aplicar criterios coherentes a la hora de entender las estrategias utilizadas incluso por el mismo traductor, puesto que las consiguientes dificultades lingüísticas y culturales no se han manejado igual en todos los ejemplos.

\section{REFERENCIAS BIBLIOGRÁFICAS}

Alpert, M. 1969. Two Spanish Picaresque Novels. Lazarillo de Tormes. The Swindler. Londres: Penguin.

Appelbaum, S., trad. 2001. Lazarillo de Tormes. Anonymous. A Dual Language Book. Nueva York: Dover Publications.

BlecuA, A., ed. 1982. La vida de Lazarillo y de sus fortunas y adversidades. Madrid: Castalia.

Brunette, L. 2000. “A Comparison of Translation Quality Assessment Practice”. The Translator 6, 2: 169-182.

Delabatista, D. 1996. Wordplay and Translation. Manchester: St. Jerome. Jerome.

France, P. 2000. Oxford Guide to Literature in English Translation. Oxford: Oxford University Press.

How, L., trad. 1917. The Life of Lazarillo de Tormes and his Fortunes and Adversities. With an Introduction and Notes by Charles Philip Wagner. Nueva York: Mitchell Kennerley. 
LutTikhuisen, F. 1997. “The Elizabethan Translators”. Actas de las I Jornadas Nacionales de la Historia de la Traducción, León 1987. Eds. J.C. SANTOYO, R. RABADÁn, T. GuzMán, J.L. Chamosa. León: Universidad de León. 177-182.

PARsons, J., trad. 1966 The Life of Lazarillo de Tormes; his Fortunes and Adversities. Introduction by Glen Willbern. Nueva York: American Corporation.

LANDERs, C., ed. 2001. Literary Translation: A Practical Guide. Clevedon, Tonawanda, North York, and Mona Vale: Multilingual Matters.

RABADÁN, R. 1991. Equivalencia y Traducción: problemática de la equivalencia translémica inglés-español. León: Universidad de León.

Redfern, W. 1984. Puns. Oxford y Nueva York: Basil Blackwell.

Rico, F. 2000. El Lazarillo de Tormes. Madrid: Cátedra.

Rowland, D., trad. 1586. The Pleasant Historie of Lazarillo de Tormes, a Spaniarde, wherein is conteined his marveilous deedes and life. Londres: Abell Ieffes.

RudDER, R. S., trad. 1973. The Life of Lazarillo de Tormes: His Fortunes and Misfortunes as told by Himself. Nueva York: Ungar Publishers.

Vergedal, J. 2000. "Propuesta analítico-evaluadora de la distorsión y sus efectos en traducción: los juegos de palabras”. Sendebar 10-11: 111-126. 